\title{
A Comparison of Active Adverse Event Surveillance Systems Worldwide
}

\author{
Yu-Lin Huang • Jinhee Moon · Jodi B. Segal
}

Published online: 15 July 2014

(C) The Author(s) 2014. This article is published with open access at Springerlink.com

\begin{abstract}
Post-marketing drug surveillance for adverse drug events (ADEs) has typically relied on spontaneous reporting. Recently, regulatory agencies have turned their attention to more preemptive approaches that use existing data for surveillance. We conducted an environmental scan to identify active surveillance systems worldwide that use existing data for the detection of ADEs. We extracted data about the systems' structures, data, and functions. We synthesized the information across systems to identify common features of these systems. We identified nine active surveillance systems. Two systems are US based-the FDA Sentinel Initiative (including both the Mini-Sentinel Initiative and the Federal Partner Collaboration) and the Vaccine Safety Datalink (VSD); two are Canadian-the Canadian Network for Observational Drug Effect Studies (CNODES) and the Vaccine and Immunization Surveillance in Ontario (VISION); and two are European-the Exploring and Understanding Adverse Drug
\end{abstract}

Electronic supplementary material The online version of this article (doi:10.1007/s40264-014-0194-3) contains supplementary material, which is available to authorized users.

Y.-L. Huang · J. Moon · J. B. Segal ( $₫)$

Department of Health Policy and Management, Johns Hopkins University Bloomberg School of Public Health, Room 644, 624

N. Broadway, Baltimore, MD 21205, USA

e-mail: jsegal@jhmi.edu

\section{J. B. Segal}

Department of Medicine, Johns Hopkins University School of Medicine, Baltimore, MD, USA

J. B. Segal

Johns Hopkins Center for Drug Safety and Effectiveness,

Baltimore, MD, USA
Reactions by Integrative Mining of Clinical Records and Biomedical Knowledge (EU-ADR) Alliance and the Vaccine Adverse Event Surveillance and Communication (VAESCO). Additionally, there is the Asian Pharmacoepidemiology Network (AsPEN) and the Shanghai Drug Monitoring and Evaluative System (SDMES). We identified two systems in the UK-the Vigilance and Risk Management of Medicines (VRMM) Division and the Drug Safety Research Unit (DSRU), an independent academic unit. These surveillance systems mostly use administrative claims or electronic medical records; most conduct pharmacovigilance on behalf of a regulatory agency. Either a common data model or a centralized model is used to access existing data. The systems have been built using national data alone or via partnership with other countries. However, active surveillance systems using existing data remain rare. North America and Europe have the most population coverage; with Asian countries making good advances.

\section{Key Points}

We systematically conducted an inventory of ongoing initiatives that use large-linked databases for active drug safety evaluation

Active surveillance systems for adverse drug events remain rare, but electronic health data are likely to increase the feasibility of active surveillance

Currently, the systems supplement existing adverse drug reaction reporting systems, by amplifying and/ or refining safety signals. Signal generation without pre-specified safety questions is uncommon 


\section{Background}

For decades, post-marketing drug safety surveillance has depended on analysis of spontaneous adverse drug events (ADEs). Systems such as the FDA Adverse Event Reporting System (FAERS) in the USA and the World Health Organization (WHO) Programme for International Drug Monitoring [1, 2] were established to improve post-marketing surveillance for ADEs. However, the system relies on voluntary reporting by healthcare professionals or patients and their families. In many countries, including the USA, the law requires pharmaceutical and medical device manufactories to report ADEs to the drug regulatory authorities [3]. These spontaneous reporting systems, nevertheless, are hampered by incomplete information in the reports, such as on the exposures or outcomes, which limit the value of the data. [4]. Additionally, the healthcare community often fails to report events with well-established causality, diminishing our ability to establish the prevalence of ADEs with passively reported data. Moreover, there is under-reporting of events that are not hypothesized to be drug related, unless the events are very severe.

Given the deficiencies inherent in systems that rely on spontaneous ADE reporting, there are many proactive approaches to study the causal relationship between medical interventions and harmful effects. For example, in the Netherlands, the Lareb Intensive Monitoring's web-based tool collects primary information from patients for pharmacovigilance [5]. Other strategies include the use of casecontrol networks to identify ADEs or hospital-based intensive monitoring systems [6]. Yet, there is ongoing interest in developing systems that can incorporate and use existing electronic data such as administrative claims and electronic health record (EHR) data to enable active surveillance for ADEs [7]. The latter method can be efficient, as it does not require the collection of new information. It enables investigators to actively query existing information, and provides a more holistic picture of drug use in a community.

Drug regulatory agencies and the pharmaceutical industry in the USA and many European countries are vigorously developing active surveillance systems for pharmaceutical products, vaccines, and medical devices [8]. We aimed to review the status of active surveillance systems for the detection of ADEs, worldwide, with the goal of informing communities considering active surveillance for ADEs in their population.

\section{Methods}

We conducted an environmental scan to identify active surveillance systems. In brief, we relied on a search of the published literature to identify citations describing active surveillance systems. We also searched online for evidence of other active surveillance systems, not detected in our published literature review. We then identified the best sources of information about these systems and extracted this information into tables. Additionally, we sought the input of experts who have been key leaders in active surveillance systems in the USA to review our list of systems and suggest missed active surveillance systems for incorporation in this review.

\subsection{Key Components}

The key components of the active surveillance systems that we sought to capture with this environmental scan were: Structure-The structure, partnership relationships, and funding of existing active surveillance systems; Data-The data used in existing surveillance systems, what patient populations are included, and what is the content of these data; Function-Examples of ADEs that have been identified with the active surveillance systems.

\subsection{Scanning Strategy}

A literature search was conducted to identify articles containing information on active surveillance systems from electronic databases PubMed, Google Scholar, and EMBASE from 2003 to 2013 on October and November 2014 (see electronic supplementary material Tables 1 and 2). We searched using MeSH terms and key word searches relevant to this topic including: "product surveillance, "postmarketing", "pharmacovigilance", "adverse drug event", "adverse drug reaction reporting systems" [MeSH Terms], and "database management systems." No language barrier was set for the query, but the abstract required a title and abstract in English. We also searched for citations describing known active surveillance systems such as the Sentinel Initiative and Exploring and Understanding Adverse Drug Reactions by Integrative Mining of Clinical Records and Biomedical Knowledge (EU-ADR). We supplemented this with searches using online search engines (Google and Bing) to identify references to active surveillance systems that we had not found in the professional literature.

\subsection{Review Strategy}

Paired authors reviewed the titles of the citations identified in the electronic search for relevance to active surveillance. If any single author thought it to be potentially relevant, it was retrieved for further review. We did not seek to identify every article that described a given active surveillance system-a single article that alerted us to the presence of the system was sufficient. We created a 


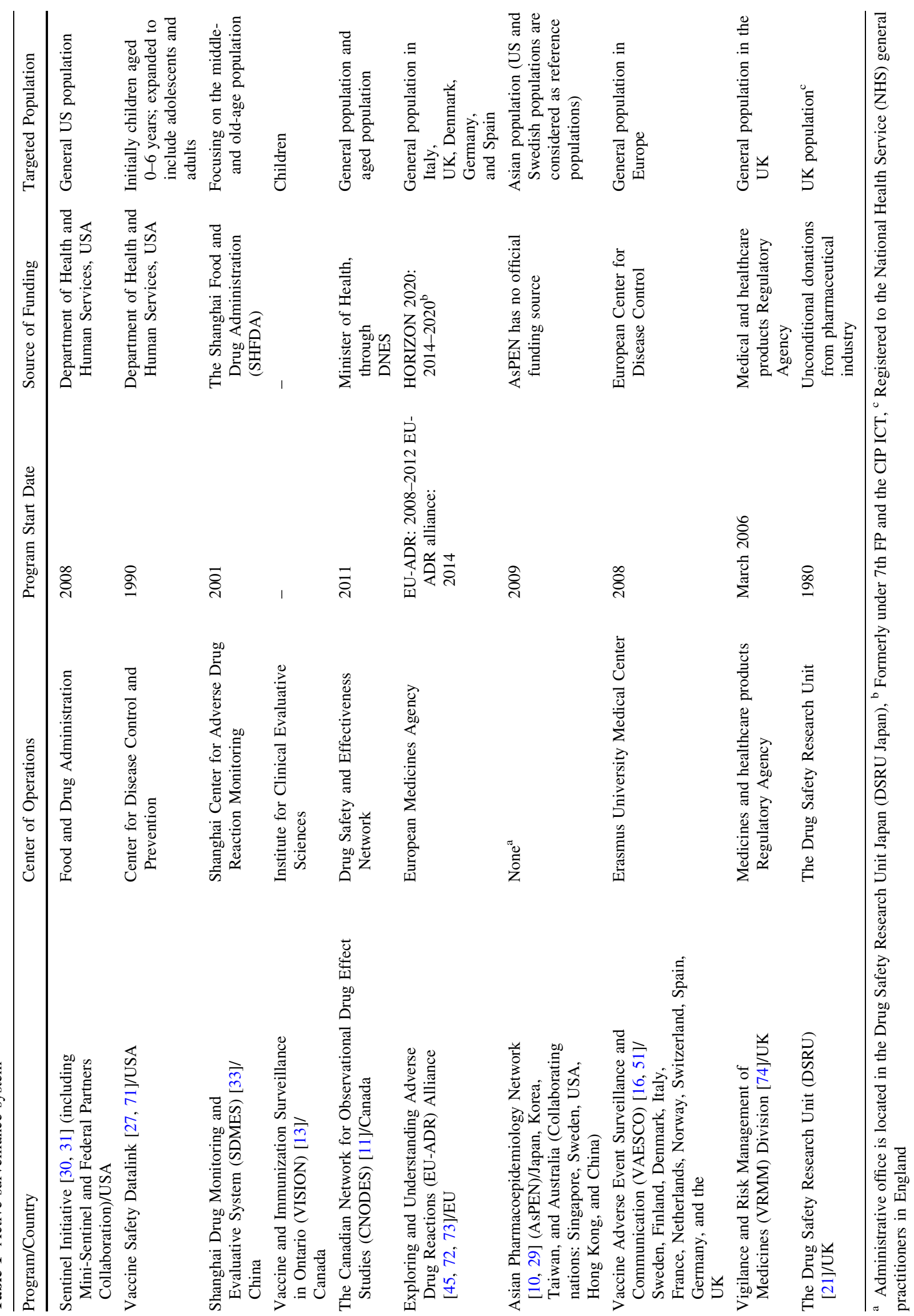




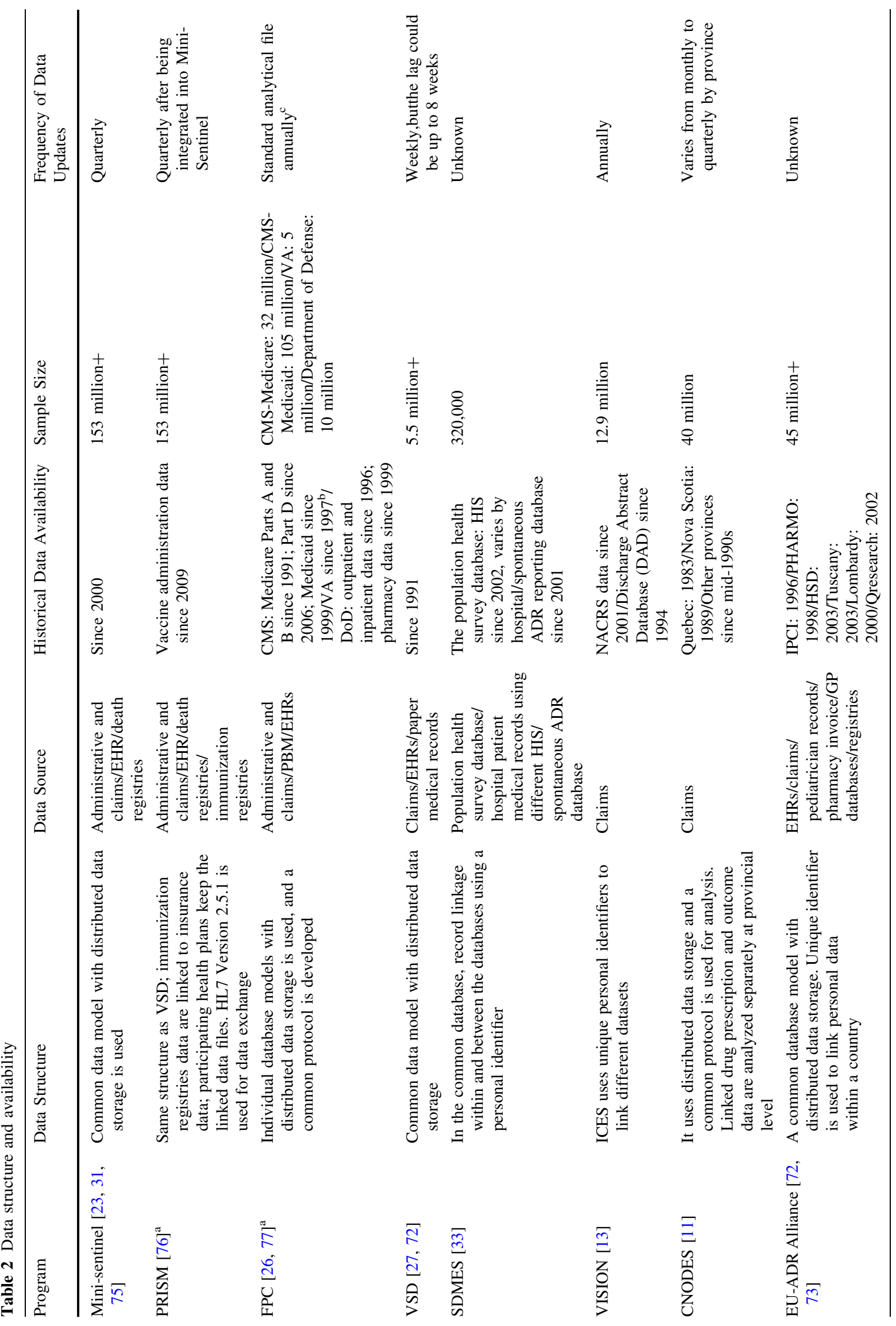




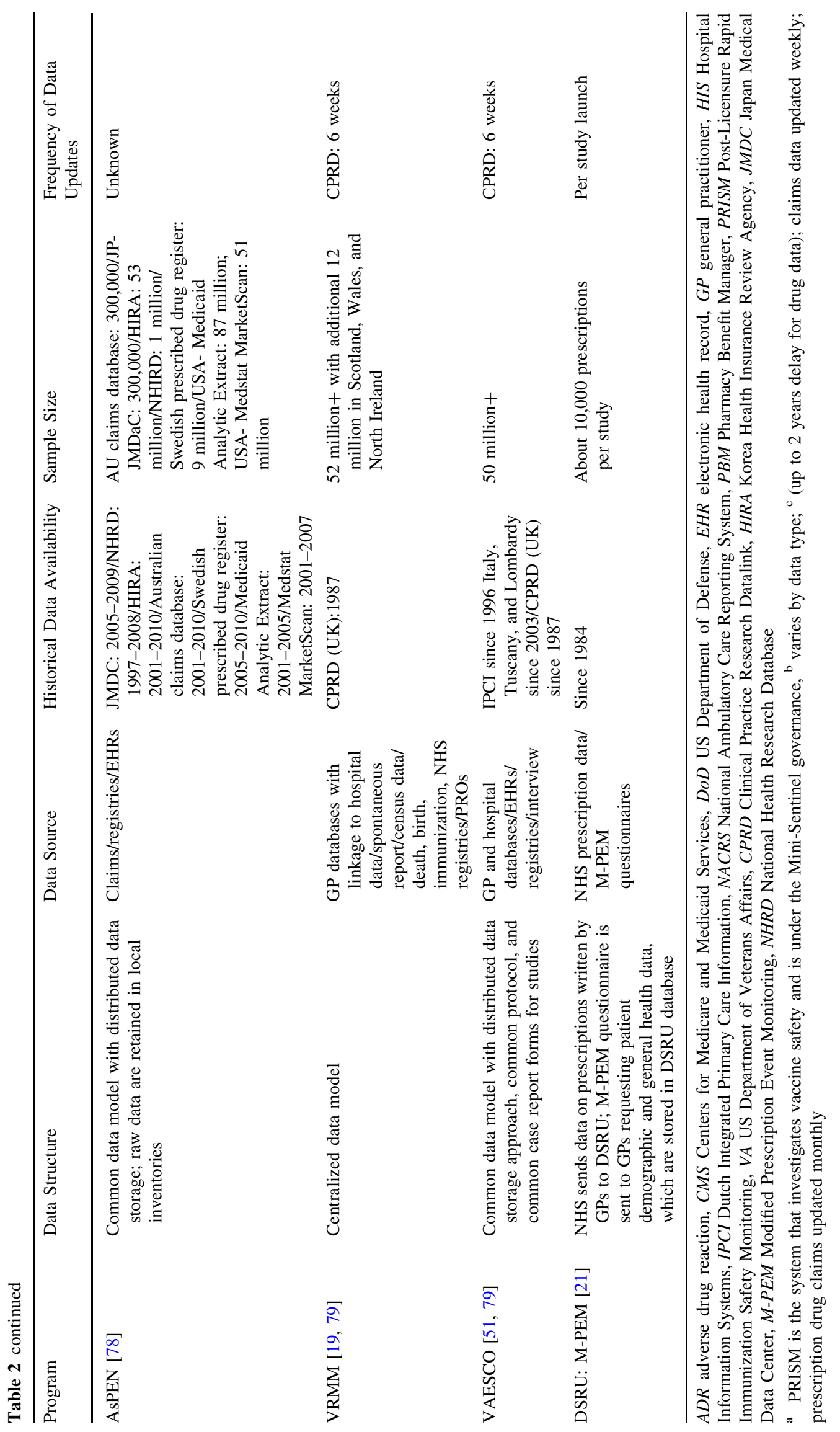


working definition for the purposes of the scanning exercise. For our review, an active surveillance system has the following features (the first four were adapted from the work by Aronson and colleagues) [9]:

- It is designed for post-marketing surveillance of pharmaceutical products (including vaccines).

- It has a goal of generating post-marketing drug safety information.

- It does not require personnel to initiate safety reports (describing individual cases).

- It uses real-world data that are generated from routine practice, requiring no direct patient contact.

- Data cannot be from a single institution.

The three authors came to a consensus about the active surveillance systems that would be described in the environmental scan based upon their meeting the criteria described above. We opted to exclude studies conducted in single institutions because we expected that these operations would not have had the challenge of integrating data from various sources, which is typical of most active surveillance systems.

\subsection{Data Extraction}

The authors identified the published articles or online sources with sufficient information to populate the data tables. Frequently, more than one source was needed to identify the relevant information. Data were extracted into table shells that were developed to describe the key components of the active surveillance systems.

\section{Results}

We identified nine active surveillance systems meeting our criteria worldwide. (Table 1) Most systems are in North America and Europe. We first provide an overview of the systems and then describe commonalities across systems.

\subsection{Regional Overview of Pharmacovigilance Systems Worldwide}

\subsubsection{Asia}

In Asia, the Shanghai Center for Adverse Drug Reaction Monitoring has led the Shanghai Drug Monitoring and Evaluative System (SDMES) since 2001. The SDMES is an evaluation and surveillance system designed for the local monitoring of marketed drugs. It works in partnership with ten hospitals in Shanghai that permit direct access to patient information.
Other countries in Australasia (Japan, Korea, Taiwan, and Australia) have taken a different approach. They formed a voluntary research network, the Asian Pharmacoepidemiology Network (AsPEN) [10]. Recently, the USA, Sweden, Hong Kong, China, and Singapore have become collaborators with AsPEN. This cross-national endeavor aims to facilitate identification and validation of emerging safety issues for pharmaceutical products across Asian countries.

\subsubsection{Canada}

In response to the Food and Consumer Safety Action Plan, which was designed to strengthen consumers' health, Health Canada formed the Drug Safety and Effectiveness Network (DSEN). The DSEN, on behalf of Health Canada, and the Canadian Institutes of Health Research (CIHR) began the Canadian Network for Observational Drug Effect Studies (CNODES) in March 2011. CNODES is tasked with coordinating drug safety and effectiveness research for drugs approved for sale in Canada [11, 12]. The CNODES established a network that brings together researchers, as well as databases, from provinces across Canada. Aside from the Canadian provincial databases, CNODES also accesses the UK's Clinical Practice Research Datalink (CPRD; formerly GPRD) as this allows CNODES to investigate drugs marketed in the UK before they are launched in Canada.

Before the establishment of CNODES, the Institute for Clinical Evaluative Sciences (ICES) created the Vaccine and Immunization Surveillance in Ontario (VISION) as a vaccine vigilance system. The ICES is a healthcare and health services research institute in Ontario, and also houses Ontario's healthcare claims data [13].

Active Surveillance System Abbreviations AsPEN: Asian Pharmacoepidemiology Network CNODES: The Canadian Network for Observational Drug Effect Studies

DSRU: The Drug Safety Research Unit

EU-ADR Alliance: Exploring and Understanding

Adverse Drug Reactions Alliance

FPC: Federal Partners Collaborative

SDMES: Shanghai Drug Monitoring and Evaluative System

VAESCO: Vaccine Adverse Event Surveillance and

Communication

VISION: Vaccine and Immunization Surveillance in

Ontario

VRMM: Vigilance and Risk Management of

Medicines Division

VSD: Vaccine Safety Datalink 


\subsubsection{European Union}

There has been important legislation regarding drug safety in Europe. In 2010, the European Union (EU) adopted new pharmacovigilance regulations that strengthened coordination and data collection-Directive 2010/84/EU and Regulation 1235/2010. Since this legislation, the European Medicines Agency (EMA) can charge a fee to pharmaceutical companies specifically for the costs of pharmacovigilance systems, and the systems can move forward providing holistic examination of drug safety.

The EMA piloted a project, EU-ADR, which implemented a computerized system to detect ADEs with extracted EHRs between 2008 and 2012[14]. It developed standardized procedures and software for extraction and aggregation of data from multiple sources in different European countries, developed methods for pooled data analysis, and established a web platform as an open workspace for the integrated analysis. Based on the system developed during the EU-ADR project, the EU-ADR Alliance emerged as a federated collaborative framework for drug safety studies, using eight European populationbased administrative and healthcare databases from Italy, Netherlands, UK, Germany, and Denmark [15].

Additionally, since 2008, the European Centre for Disease Prevention and Control (ECDC) has funded a consortium of researchers in EU countries. This supported VAESCO: Vaccine Adverse Event Surveillance and Communication (VAESCO), which was established to study ADEs following immunization [16, 17]. VAESCO's most recent activity was in 2012 when it completed a project studying narcolepsy after influenza vaccines.

\subsubsection{United Kingdom}

The UUK has two active systems: the Vigilance and Risk Management of Medicines (VRMM) and the Drug Safety Research Unit (DSRU). As part of Medicines and Healthcare products Regulatory Agency, the VRMM maintains ongoing activities to monitor the safety of marketed medicines [18]. VRMM is also involved in pharmacoepidemiology, research and intelligence, and benefit-risk review [18]. VRMM has implemented proactive pharmacovigilance by the expert use of CPRD-the large-linked observational datasets from a range of primary and secondary care settings. This along with its "Yellow Card" scheme, a spontaneous reporting system, allows it to conduct a comprehensive review of selected healthcare products [19].

The DSRU was founded in 1980 as an independent academic unit and has been conducting post-marketing surveillance, pharmacoepidemiology, and risk management studies across Europe. It uses diverse European data for pharmacovigilance, but predominantly data from the UK. Investigators in the DRSU developed a novel method for early detection of unrecognized drug hazards of newly marketed drugs, known as prescription-event monitoring (PEM) [20]. In the late 1990s, DSRU established an updated version of PEM, Modified PEM (M-PEM), which surveys general practitioners. For their investigations, they target a sample of 10,000 patients prescribed a given drug, and link prescription data to census data and healthcare databases [21].

Although the DRSU is fundamentally different than the other systems we included in this review, the M-PEM method of DSRU met our criteria for active surveillance. The process requires that general practitioners complete questionnaires and send them back to DRSU; however, this is a systematic process employed when there is a suspected adverse event (this is not case reporting). General practitioners are randomly selected to respond to questionnaires. We consider this to be active surveillance because nearly all UK residents are registered to general practitioners and data on nearly all prescriptions issued by general practitioners are sent by pharmacists to the Prescription Pricing Authority, which is responsible for reimbursement. Because DSRU targets all prescriptions that were issued since the launch of the drug of interest, it can be considered as an active post-marketing surveillance system.

\subsubsection{USA}

In response to the Food and Drug Administration Amendment Act of 2007, the US FDA has collaborated with public, academic, and private entities to develop a national electronic safety monitoring system, the Sentinel Initiative. Several projects were initiated under the Sentinel Initiative to augment existing safety monitoring systems. The Foundation for the National Institutes of Health launched the Observational Medical Outcomes Partnership (OMOP) in late 2008 as a 2-year pilot project to identify the needs of the Sentinel Initiative and to develop the essential methods and data infrastructure to allow the reuse of automated healthcare data [22].

Harvard Pilgrim Health Care Institute coordinated a pilot project, the Mini-Sentinel. The Mini-Sentinel's objective is to develop the framework, data resources, analytic capabilities, policies, and procedures that will be required for a fully operational Sentinel System [23]. In addition to pharmaceutical product surveillance, the MiniSentinel also includes the Post-Licensure Rapid Immunization Safety Monitoring (PRISM) project and the Blood Safety Continuous Active-Surveillance Network Feasibility Evaluation (Blood-SCAN) project. Blood-SCAN fulfils the regulatory need for maintaining a safe blood supply [24]. Although the Mini-Sentinel Initiative officially ended in September 2014, the contract was been changed to be a new 5-year cooperative agreement. Furthermore, a new 
public-private partnership, the Innovation in Medical Evidence Development and Surveillance (IMEDS), was established to build on the work of the Mini-Sentinel and OMOP to enhance post-marketing pharmacovigilance and facilitate the use of EHRs [25].

The FDA also cooperates with Veteran's Affairs, the Department of Defense, and the Centers for Medicare and Medicaid Services on the Federal Partner Collaboration (FPC), which provides access to federally owned healthcare data [26]. This collaboration allows the FDA to assess the safety and effectiveness of products in unique and potentially vulnerable populations.

The VSD predates the Sentinel Initiative. Since 1990, the VSD, managed by the Centers for Disease Control and Prevention (CDC), has been providing evidence, particularly about safety, to inform national immunization policy through population-based research [27]. With more than 2 decades of surveillance, the VSD has repeatedly demonstrated its ability to investigate and detect safety signals, and evaluate vaccine effectiveness [28].

\subsection{Structure of the Active Surveillance Initiatives}

The initiatives described above are mostly research networks or institutes; some are public-private collaborations. Only the VRMM (UK) and the SDMES (China) are administered through government agencies. However, nearly all receive funding from public sources (Table 1). Only AsPEN (Asia) does not have an identifiable funding source [29]; the DSRU (UK) is the only one that receives funding mainly from the private sector.

Each system has a somewhat different operational structure. DSRU (UK) is a registered independent charity that receives support from the National Health Service (NHS) Business Service Authority. The DSRU also receives unconditional donations from pharmaceutical companies. The DSRU has authority to access and uses patient-identifiable data without patient consent based on the permission from the Ethics and Confidentiality Committee of the NHS National Information Governance Board.

The USA took a public-private approach to data. Because there is no universal public insurance coverage, both the Sentinel Initiative and VSD cooperate with private insurers as data partners. The CDC's Immunization Safety Office partners with managed care organizations throughout the USA to allow the VSD to rapidly detect adverse events after immunization [27].

In the Sentinel Initiative's Mini-Sentinel Pilot (USA), Harvard Pilgrim Health Care Institute serves as the coordinating center and collaborates with multiple private insurance plans as data partners $[30,31]$. The Sentinel Initiative's Federal Partners Collaborative includes the Center for Medicare and Medicaid Services, the Veterans
Administration, and the Department of Defense, which allows this Collaborative to query federally held, electronic healthcare data.

Many initiatives adopt a collaborative approach by engaging researchers from multiple institutes such as the EU-ADR Alliance (EU), the CNODEs (Canada), and the AsPEN (Asia). The approach may be an efficient and flexible way to initiate safety studies. In the mechanisms of CNODES and EU-ADR Alliance, the regulatory authorities (EMA and Health Canada) pose study questions regarding drug safety and effectiveness to the study coordinating office. In CNODES, other federal agencies and health plans can submit their questions to Health Canada [11]. Then, the scientific advisory committee evaluates and sets up a research agenda, and a project team is formed to answer the question. In the AsPEN, participating researchers achieve census on the pressing study questions regarding drug safety [10], and then teleconferences are held to develop protocols and to decide on the study execution details.

\subsection{Data}

The initiatives generally rely on existing population-based data; most use administrative claims (Fig. 1; Table 2). Many also have the ability to link to medical records. Death and cancer registries are anonymously linked within the provinces in Canada [13]. The source of exposure data for the VSD (USA) comes from the automated systems of managed care organizations that track immunizations administered to members [32]. For evaluating vaccine safety, all of the vaccine surveillance systems link medical records to vaccine registries.

The large-linked databases have rich information (Fig. 2). The databases contain demographic information and prescription information. Except for the SDMES (China), the other initiatives can capture longitudinal information on medical use from outpatient records effectively, but are less able to aggregate inpatient data. The SDMES (China) uses medical records directly from hospitals, but cannot access outpatient information. Software is installed in each hospital's information system to capture required information, and information is sent to the SDMES center periodically [33]. The CPRD used by the VRMM (UK) allows for the tracking of medical activity and drug use seamlessly. Because of the rich information contained in EHRs, most initiatives are actively working to capture and incorporate this into their data, so that they have laboratory results and richer clinical information than can be known from claims data.

Legal and privacy has been an important concern in many countries, such as the Health Insurance Portability and Accountability Act (HIPAA) in the USA. Most 

in worldwide active surveillance systems. The Federal Partners Collaboration and Mini-Sentinel are counted separately, and systems may use multiple types of databases. EHR electronic health records, $P B M$ pharmacy benefits manager
Fig. 1 Types of databases used

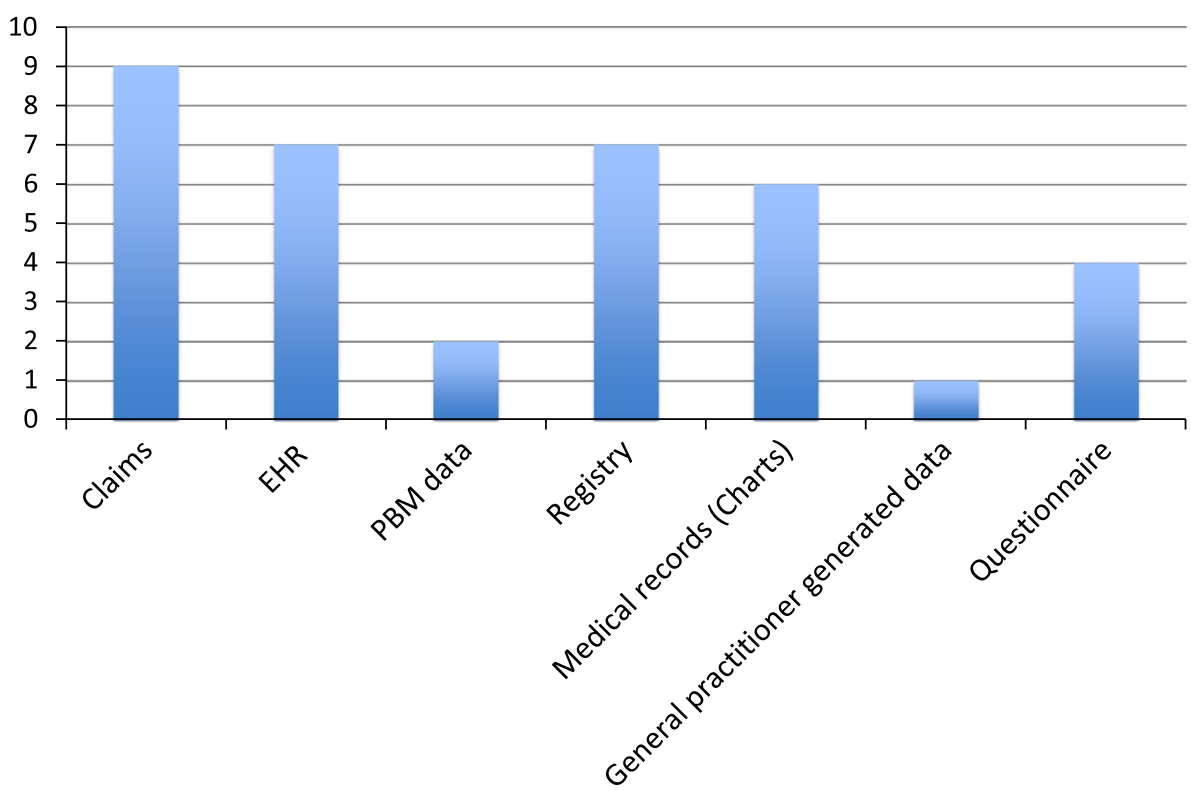

Fig. 2 Characteristics of data elements used in worldwide surveillance systems. The

Federal Partners Collaboration and Mini-Sentinel are counted separately. The percentage of each data element is calculated based on available data in each category. Additionally, vaccination surveillance systems were not calculated in "drug codes" and "quantity"

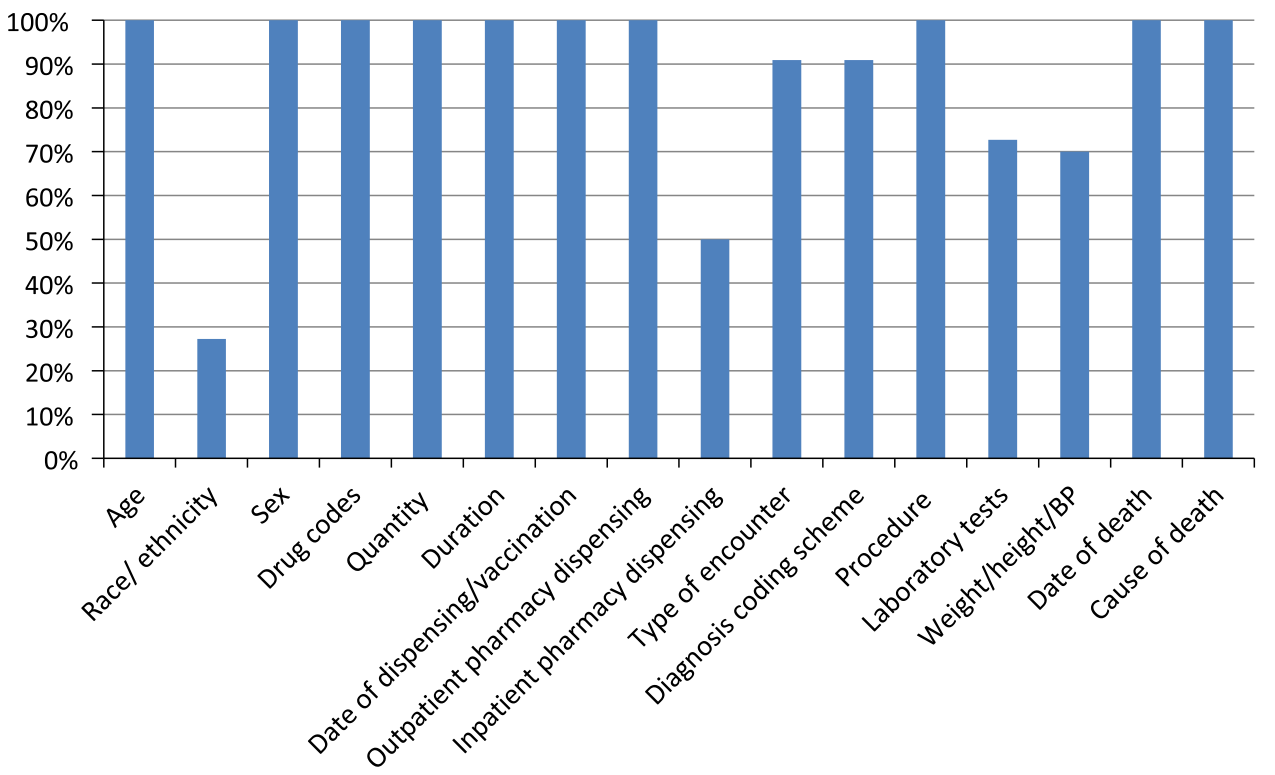

initiatives choose not to pool data directly from multiple databases in a single central warehouse. Most initiatives chose a distributed data network approach, meaning most data remain in situ, and data partners have ownership and physical control of their protected data. In many initiatives, data are owned by different entities, for example, in the Mini-Sentinel (USA), data are owned by the different health plans; in the EU-ADR Alliance, data are owned by either government agencies or research institutes. Because of the concerns about the control of data, the VSD (USA) moved from using a central repository of data to distributed data storage [34]. Distributed data storage also facilitates collaboration across countries as is seen in the AsPEN (Asia) and the EU-ADR Alliance.
To aggregate data across sites, many initiatives use a common data model or common protocol. In CNODES (Canada) and the Federal Partner Collaborative (USA), common protocols are developed to standardize data queries and analyses in each database. Local content experts remain involved throughout the process. In CNODES, content experts are included in each project team [11].

The Mini-sentinel (USA), EU-ADR (EU), AsPEN (Asia), and VSD (USA) have developed their own common data models so that they can standardize data queries and perform analyses locally with pre-specified statistical packages. The common data model consists of separate tables, each of which stores a specific type of data 
generated from a query. Using a common data model, standardized common input files are generated locally by each data partner. An internal identifier is included in the input files so that each unique patient can be linked across local files, providing a comprehensive medical history during the period the patient receives care from that institution or is enrolled in that insurance plan. The coordinating centers typically develop standard statistical packages that are distributed to each site that contributes data. The aggregated, de-identified, and encrypted files, which include patient, drug, and event information, are sent to a central repository for further evaluation and analysis. Sometimes, only the results of local analyses are transmitted centrally. Jerboa ${ }^{\circledR}$, used by the EU-ADR Alliance, is a custom-built JAVA software developed to query and analyze local patient data [14].

Initiatives with smaller populations, such as VISION (Canada) and SDMES (China), have chosen central data models. Moreover, the methods of data collection for the CPRD (from general practitioners in the UK) necessarily make these central databases. There are several methods to link patient data in a central model. If they are used across multiple systems, the most straightforward way is to use deterministic linkage, based on unique personal identifiers. The SDMES and CPRD use this approach to generate comprehensive patient-level histories. The CPRD uses the universally adopted NHS number to link patients' data from general practitioners to hospital medical records. Another approach is to use probabilistic record linkage, which is adopted by one of the EU-ADR Alliance databases, the PHARMO database. The probabilistic record linkage uses Bayesian likelihood estimations and learningbased rules to estimate the likelihood that two files belong to the same person [35].

Unlike other systems that use EHRs or claims data, the DSRU (UK) collects prescription information from the government and collects questionnaire-based responses from primary care doctors in their data warehouse. This approach incorporates both retrospective and prospective data collection.

For initiatives that involve multi-institutional collaboration, there are assorted data harmonization processes in place. For example, the EU-ADR Alliance uses databases having different characteristics, so the Unified Medical Language System (UMLS) is used to map different terminologies [36]. The PRISM (in the US Mini-Sentinel) chose to use the HL7 system and applied this to acquire standardized information from different data partners.

In some cases, use of a distributed data network delays access to data if there are many involved sites. In the FPC (USA), claims data are updated weekly, and prescription drug claims are updated monthly. However, it may take up to 2 years for drug data in the standard analytical files of the FPC to be available to the research community [37]. The VSD (USA) and Mini-Sentinel (USA) can refresh data files weekly and quarterly, respectively. The VSD developed a real-time surveillance system and initiated its use in an ongoing study of a new meningococcal vaccine for adolescents [38]. The VSD's rapid cycle analysis approach uses relatively simple structured and aggregated data for weekly analyses; data are segmented into weekly cohorts of vaccinated children. More accurate data are then available after 8 weeks.

\subsection{Function-Examples of Safety Events Evaluated}

Currently, all of the initiatives investigate safety questions in response to regulatory agencies' requests. Active surveillances play supportive roles to spontaneous systems and do not aim to replace them. Most surveillance systems see their role as signal refinement rather than signal detection, although the vaccine ADE systems aim to detect safety signals (Table 3 ).

Maximized sequential probability ratio testing (maxSPRT) is one of the commonly used techniques and has been used in the VRMM's (UK) enhanced proactive pharmacovigilance, as well as by the Mini-Sentinel and the VSD (USA) [39]. MaxSPRT supports continuous or timeperiod analyses as data are collected, and allows detection of a change in the probabilities of ADEs after the introduction of a new vaccine or drug [38]. The VSD has used maxSPRT to analyze its weekly updated data for real-time surveillance since 2005 [40]. VSD has studied the association between quadrivalent human papillomavirus vaccine and venous thromboembolism [41].

The Mini-Sentinel team developed the Cohort Matching Prospective Routine Observational Monitoring Program Tool (PROMPT) module, which is a suite of modular SAS macros that enables effect estimation in distributed data, using a propensity-score-matched sequential cohort of new users of the drug under investigation [42]. It allows the FDA to investigate prespecified health outcomes in newly marketed pharmaceutical and biological products. MiniSentinel investigators have been monitoring a wide range of products; an example is the surveillance for ischemic stroke, intracranial hemorrhage, and gastrointestinal bleeding in new users of rivaroxaban compared with users of warfarin [43].

The VRMM (UK) has developed methods for near realtime sequential analysis of ADE reports that come in from its Yellow Card scheme. Epidemiological analyses using the CPRD are then used to refine and evaluate these safety signals [19]. The team has evaluated the association between bivalent human papillomavirus vaccine (Cervarix) 


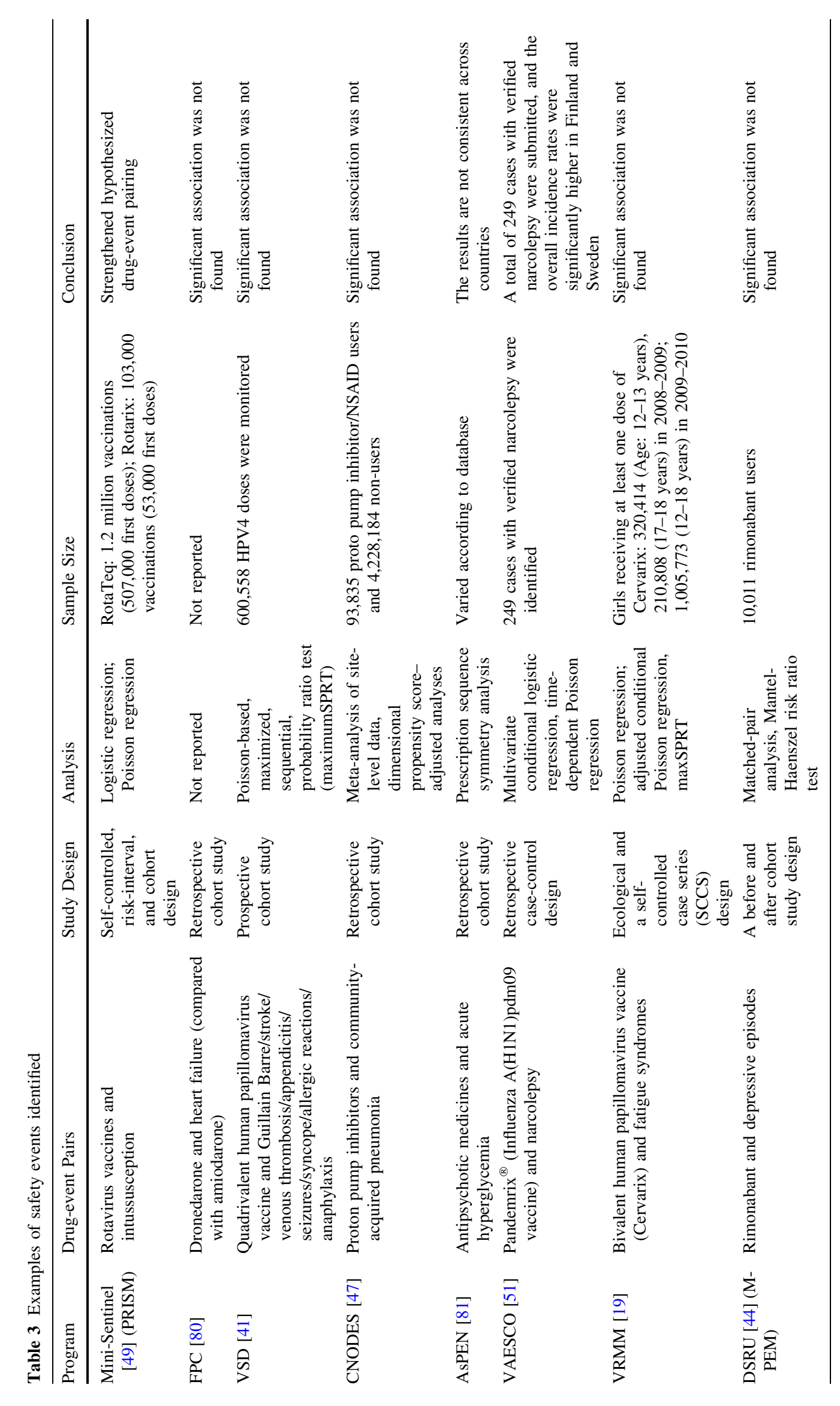


and fatigue syndromes with an ecological and a self-controlled case series design [19]. The DSRU (UK) using its M-PEM technique evaluated the safety of rimonabant, which was suspected of causing depressive episodes. By comparing the risk of major and minor depressive episodes before and after initiation of rimonabant, DSRU concluded that rimonabant causes depressive episodes in patients without a previous psychiatric history [44].

Disproportionate reporting is a methodology use by EudraVigilance, part of the EU-ADR Alliance. Based on a signal detected with disproportionate reporting analyses, the EU-ADR Alliance developed a research protocol to investigate the association between risk of cardiac valve disorders and the use of bisphosphonates, in response to EMA's request [45]. Main analyses employed data mining techniques to explore the association with automated matched case-control methods [45].

CNODES (Canada) developed a research protocol according to Health Canada's request to evaluate the association between the use of high-potency statins and acute kidney injury [46]. This study used a nested casecontrol analysis with propensity-score adjustment. Another study testing the association between proton pump inhibitors and pneumonia used high-dimensional propensity scores to match exposed and control groups [47]. Cohort, case-control, risk-interval, and self-controlled case-series designs are often used to assess vaccine safety [48]. The VISION (Canada) researchers have used the linked data for self-controlled case-series analyses to investigate the association between vaccination and emergency room visits, hospital admissions, and deaths [13].

The Mini-Sentinel (USA) has investigated the association between rotavirus vaccination and intussusception with a self-controlled risk-interval and cohort design in its PRISM program [49]. With access to data on so many exposed individuals, the research team had sufficient power to answer the FDA's query. The FDA later required revised labeling for RotaTeq ${ }^{\circledR}$ [50]. VAESCO (EU) studied the safety of Pandemrix ${ }^{\circledR}$, a monovalent 2009 H1N1 influenza vaccine [51]. The researchers chose a retrospective casecontrol design. As the results could not demonstrate a strong association between vaccination of Pandemrix ${ }^{\circledR}$ and narcolepsy, the EMA concluded that the benefits still outweigh the risk with Pandemrix ${ }^{\circledR}[52,53]$.

\subsection{Limitations in Current Initiatives}

Accurate identification of adverse health outcomes and medicine use is critical in any active surveillance systems. All of the initiatives largely use existing data, which means the data are not collected primarily for research purposes. Therefore, each initiative needs to take steps to ensure the validity of coding. The Mini-Sentinel, CPRD, and EUADR have made great efforts to validate algorithms to identify health outcomes of interest in given datasets [5458]. The researchers have used external sources to confirm health outcomes, but the validity varies widely. In the Mini-Sentinel, investigators found positive predictive values in the range of $25 \%$ (for liver disease) to $66 \%$ (for anaphylaxis); in CPRD, confirmed diagnoses among studies were in the range of 24-100\%.

Many initiatives that use EHR data have limited ability to analyze very new products. Part of the success of the Mini-Sentinel project came from learning from vaccine surveillance. However, there are significant differences between surveillance of vaccines and of drugs. One important factor is the time for diffusion of use of new products into the market. Vaccines are often funded by the public sector and made widely available so there is less difficulty in having enough exposed individuals to study. Because most of the systems use insurance healthcare databases, delayed uptake of new drugs owing to lack of coverage or formulary restrictions, means that sufficient users of a new medication may not be available for study.

Although some initiatives are using EHR data, the overall use of EHR is low; most of the data being used are from administrative claims. Thus, the ability to study outcomes and ADEs that require analysis of laboratory results is limited. Detection of ADEs such as liver injury is challenging with claims data alone.

The Sentinel Initiative, EU-ADR, and AsPEN employed a common data model to aggregate data, but use of a common data model may result in some loss of data integrity. When researchers tested the OMOP common data model in the THIN database, they mapped medical and drug codes. Twenty-five percent of the diagnosis codes and $55 \%$ of the drug codes in the raw THIN database were not retrieved with the OMOP terminology dictionary [59]. Continuing work is needed to improve application of algorithms across different data sources.

Active surveillance is a resource-intensive task. Active surveillance systems require advanced information technology infrastructure to store and process analyses. The EMA and pharmaceutical industry contributed 6 million Euros to the 4-year EU-ADR project [60]. The FDA awarded a 5-year contract to the Harvard Pilgrim Health Care Institute to carry out the Mini-Sentinel pilot project for 72 million US dollars [61]. The New Zealand Pharmacovigilance Centre at the University of Otago operated the Intensive Medicines Monitoring Programme for PEM, and has been suspended because of a lack of funding [62]. The case needs to be made that there is a public health value to these systems and this needs to be demonstrated with data. 


\section{Discussion}

\subsection{Summary of Findings}

A number of review articles have discussed individual systems separately [23, 32, 63, 64], our report may be the first to systematically describe the worldwide, active drug surveillance systems that use existing data. We have found that while databases of healthcare information are increasingly prevalent, there are relatively few countries involved in active surveillance and these are largely in North America and Europe. We identified no systems that cover medication-exposed patients in India, the Eastern European countries, or the South American and African continents. Other systems are early in development and are promising. We propose that the most successful systems are those able to accurately and efficiently capture prescription and use data, can refresh information frequently, have easy access to experts for advanced statistical analyses, and have a large patient population from which to draw data.

There is no single way to success. Diverse approaches are taken to conduct active surveillance because of different healthcare systems. We note that the VSD (USA) is the oldest system and has decades of experience in active postmarketing vaccine surveillance; this has importantly informed the development of later systems. The VRMM (UK) has, perhaps, the greatest ability to capture medical use comprehensively, because of their use of the longestablished and very rich CPRD data. The centralized model adopted by the UK, the decentralized model adopted by the USA, and the research network approach adopted by Canada are valuable models of different approaches that might be taken to accomplish ADE surveillance. Similarly, the innovative collaborative model of AsPEN (Asia) is another approach to regional pharmacovigilance, which addresses participating countries' needs.

\subsection{Other Initiatives}

We note that there have been other endeavors to detect safety signals without relying on spontaneous reports; these include the Immunogenicity Surveillance Registry of Erythropoiesis Stimulating Agents in Thailand, the North American Antiepileptic Drug Pregnancy Register in the USA, and activities with the Australian Rheumatology Association Database [65-67]. These did not meet our criteria for active surveillance systems, but have many similar characteristics. Large-linked databases have appropriately gained attention given their tremendous potential for safety surveillance. An example is the open source initiative, the Observational Health Data Sciences and Informatics (OHDSI), which is housed at Columbia University [68]. This multi-stakeholder interdisciplinary collaborative aims to enhance the value of observational health data by using large-scale analytics. OHDSI applies methods to observational data aimed at answering realworld clinical questions, including about drug safety. There are many similar examples.

Another system with great similarities to those reviewed here is the Medical Information for Risk Assessment Initiative (MIHARI) initiated by the Japanese government with the Pharmaceuticals and Medical Devices Agency. The MIHARI project aims to develop a system that can access electronic health information from different sources, develop pharmacoepidemiological methodology for evaluating ADEs, explore existing healthcare databases for pharmacovigilance, and create a central data database with linked electronic health information and a common data format such as HL7 [69]. When implemented, we expect that this will meet our criteria for active surveillance, but it is not yet fully functional.

The EMA also carries out two initiatives that are not pharmacovigilance systems but support the pharmacovigilance activities of the EU-ADR. The Pharmacoepidemiological Research on Outcomes of Therapeutics by a European ConsorTium (PROTECT) program and the European Network of Centres for Pharmacoepidemiology and Pharmacovigilance (ENCePP) project both aim to strengthen capacity for post-authorization drug safety research.

\subsection{Limitations and Strengths}

Many initiatives are still developing and growing quickly. Some systems have limited public information; extensive information about the systems could not easily be known. Although the Sentinel System provides extensive detail about their data model, this was diffcult to obtain from most of the other systems. We collected data from multiple sources and used a systematic approach for deciding which systems would and would not be described. We used established criteria and a protocol for determining what would be considered an active surveillance system. We searched not only for published literature but also reviewed the national regulatory agencies' official websites. Moreover, all information was reviewed by two authors to ensure the reliability of the data extracted.

\subsection{Future Research}

Systems that use automated healthcare data to enhance drug safety evaluation are developing rapidly. Hence, it is necessary to re-evaluate the development of active surveillance systems frequently. More global efforts are needed to build capacity and facilitate a proactive approach to ADE evaluation [70]. A collaborative platform, such as 
what the WHO Collaborating Centre for International Drug Monitoring-Uppsala Monitoring Centre uses for spontaneous reporting, could be set up so that stakeholders from industry, regulators, and academia are able to collaborate on pharmacovigilance using existing healthcare data.

Universal data standards such as HL7 could improve data integration and help research activity to move beyond its current scope. Developing new methodology is critical for the analysis and interpretation of large realworld data. Current initiatives are not yet entirely ready to perform data mining and signal generation through automatic process. Presently, most tasks done with large data are signal refinement or evaluation and hypothesis testing.

\subsection{Conclusions}

Our paper provides an inventory of ongoing initiatives exploring large-linked databases worldwide for actively collecting data for drug safety evaluation. Currently, the systems are mostly used to supplement existing ADE reporting systems by amplifying or refining safety signals. Signal generation without a pre-specified safety research question is not yet readily available. Fast-growing informatics capacities will strengthen the ability of researchers to evaluate drug safety after licensing.

North America and Europe have the most developed systems and most coverage of the population, owing to developed healthcare and information systems. Although most computerized databases were not established for research purposes, they have been widely used in healthcare research. Published research shows that active surveillance can be an important component of drug safety evaluation. However, methodology for this area is still developing. Hence, caution is needed when interpreting the result from real-world data given all of the inherent biases when using observational data. There are challenges with using healthcare databases for ADE surveillance, but the prospect of using these systems to support post-marketing regulatory decision making is promising. A global safety strategy with a life-cycle, risk management model becomes more and more feasible.

Acknowledgments Funding: Pilot grant funds from Sun Yat-Sen University, Guangzhou, Guangdong Province, China to the Johns Hopkins University School of Medicine.

Conflict of interest Yu-Lin Huang, Jinhee Moon, and Jodi B Segal have no conflicts of interest that are directly relevant to the content of this study.

Open Access This article is distributed under the terms of the Creative Commons Attribution Noncommercial License which permits any noncommercial use, distribution, and reproduction in any medium, provided the original author(s) and the source are credited.

\section{References}

1. Brewer T, Colditz GA. Postmarketing surveillance and adverse drug reactions: current perspectives and future needs. JAMA. 1999;281:824-9.

2. Lindquist M, Edwards IR. The WHO Programme for International Drug Monitoring, its database, and the technical support of the Uppsala Monitoring Center. J Rheumatol. 2001;28:1180-7.

3. Department of Health and Human Services/US Food and Drug Administration. IND Safety Reports. 21 The Code of Federal Regulations. 312.32; 2013.

4. Hazell L, Shakir SA. Under-reporting of adverse drug reactions: a systematic review. Drug Saf. 2006;29:385-96.

5. Harmark L, van Grootheest K. Web-based intensive monitoring: from passive to active drug surveillance. Expert Opin Drug Saf. 2012;11:45-51.

6. Organization WH. The importance of pharmacovigilance. Geneva: World Health Organization; 2002.

7. Chen RT, DeStefano F, Davis RL, et al. The Vaccine Safety Datalink: immunization research in health maintenance organizations in the USA. Bull World Health Organ. 2000;78:186-94.

8. Trifiro G, Patadia V, Schuemie MJ, et al. EU-ADR healthcare database network vs. spontaneous reporting system database: preliminary comparison of signal detection. Stud Health Technol Inf. 2011;166:25-30.

9. Aronson JK, Hauben M, Bate A. Defining 'surveillance' in drug safety. Drug Saf. 2012;35:347-57.

10. Andersen M, Bergman U, Choi NK, et al. The Asian Pharmacoepidemiology Network (AsPEN): promoting multi-national collaboration for pharmacoepidemiologic research in Asia. Pharmacoepidemiol Drug Saf. 2013;22:700-4.

11. Suissa S, Henry D, Caetano P, et al. CNODES: the Canadian Network for Observational Drug Effect Studies. Open Med. 2012;6:e134-40.

12. Health Product Vigilance 2012; http://www.hc-sc.gc.ca/dhp-mps/ pubs/medeff/_fs-if/2012-hpvf-cvps/index-eng.php. Accessed 31 Jan 2014.

13. Wilson K, Hawken S, Kwong JC, et al. Vaccine and Immunization Surveillance in Ontario (VISION): using linked health administrative databases to monitor vaccine safety. Vaccine. 2012;30:6115-20.

14. EU-ADR Final Report. http://www.euadr-project.org/drupal/files/ pdf/FinalPubishableSummary.pdf. Accessed 29 Dec 2013.

15. The EU-ADR Alliance: a federated collaborative framework for drug safety studies. http://www.euadr-project.org/drupal/files/ pdf/EU-ADR-alliance.pdf. Accessed 29 Dec 2013.

16. ECDC in collaboration with the VAESCO consortium to develop a complementary tool for vaccine safety monitoring in Europe. Euro Surveill. 2009;14:1.

17. Vaccine Adverse Event Surveillance and Communication. http:// vaesco.net/vaesco.html. Accessed 28 Jan 2014.

18. MHRA: Our Divisions. 2013; http://www.mhra.gov.uk/Aboutus/ Ourstructure/OurDivisions/index.htm. Accessed 8 Jan 2014.

19. Donegan K, Beau-Lejdstrom R, King B, et al. Bivalent human papillomavirus vaccine and the risk of fatigue syndromes in girls in the UK. Vaccine. 2013;31:4961-7.

20. Mann RD. Prescription-event monitoring: recent progress and future horizons. Br J Clin Pharmacol. 1998;46:195-201.

21. Layton D, Hazell L, Shakir SA. Modified prescription-event monitoring studies: a tool for pharmacovigilance and risk management. Drug Saf. 2011;34:e1-9.

22. Stang PE, Ryan PB, Racoosin JA, et al. Advancing the science for active surveillance: rationale and design for the Observational Medical Outcomes Partnership. Ann Intern Med. 2010;153: $600-6$. 
23. Platt R, Carnahan RM, Brown JS, et al. The U.S. Food and Drug Administration's Mini-Sentinel program: status and direction. Pharmacoepidemiol Drug Saf. 2012;21(Suppl 1):1-8.

24. Baker M, Nguyen M, Mullersman J, et al. PS3-6: Blood safety continuous active-surveillance network feasibility evaluation (Blood-SCAN). Clin Med Res. 2013;11:169.

25. Foundation R-U. Innovation in Medical Evidence Development and Surveillance (IMEDS). 2013; http://omop.org/sites/default/ files/RUF_IMEDS_060613.pdf Accessed 28 May 2014.

26. Robb MA, Racoosin JA, Worrall C, et al. Active surveillance of postmarket medical product safety in the Federal Partners' Collaboration. Med Care. 2012;50:948-53.

27. Centers for Disease Control and Prevention: Vaccine Safety Datalink (VSD). http://www.cdc.gov/vaccinesafety/activities/vsd. html. Accessed 13 Dec 2013.

28. Baggs J, Gee J, Lewis E, et al. The Vaccine Safety Datalink: a model for monitoring immunization safety. Pediatrics. 2011;127 (Suppl 1):S45-53.

29. Natasha CC, Setoguchi S. AsPEN Association Study II: introduction and overview. 2011; http://www.cnjpe.org/UploadFile/ 2011\%E4\%BC\%9A\%E8\%AE\%AE/PlenarysessionIV/PlenarysessionIV_(3-1)SokoSetoguchi_AsPENAssociationStudyII_SS.pdf. Accessed 20 Jan 2014.

30. Collaborators in Mini-Sentinel. http://mini-sentinel.org/about_us/ collaborators.aspx. Accessed 14 Jan 2014

31. Mini-Sentinel_Data_Core. Mini-Sentinel Distributed DatabaseYear 2 Summary Report. 2012; http://www.mini-sentinel.org/ work_products/Data_Activities/Mini-Sentinel_Year-2-DistributedDatabase-Summary-Report.pdf. Accessed 11 Dec 2013.

32. Davis RL, Kolczak M, Lewis E, et al. Active surveillance of vaccine safety: a system to detect early signs of adverse events. Epidemiology. 2005;16:336-41.

33. Du W, Levine M, Wang L, et al. Building a structured monitoring and evaluating system of postmarketing drug use in Shanghai. Can J Clin Pharmacol. 2007;14:e40-4.

34. Brown J, Lane K, Moore K, Platt R. Defining and evaluating possible database models to implement the FDA Sentinel Initiative. US Food and Drug Administration. 2009; http://healthne wsdaily.elsevierbi.com/ /media/Images/Publications/Archive/ TheGraySheet/35/020/01350200013/sentinel_database_models_ 05_09.pdf. Accessed 20 Dec 2013.

35. Strom BL, Kimmel SE, Hennessy S. Pharmacoepidemiology. 5th ed. Chichester: Wiley-Blackwell; 2012.

36. Avillach P, Coloma PM, Gini R, et al. Harmonization process for the identification of medical events in eight European healthcare databases: the experience from the EU-ADR project. J Am Med Inf Assoc. 2013;20:184-92.

37. Racoosin J. Conducting real-time safety evaluation with medicare data: learnings from the SafeRx Project. 2010; http://www. brookings.edu/ /media/events/2010/10/04saferx/fdaslidesround table100410final2.pdf. Accessed 15 Jan 2014.

38. Lieu TA, Kulldorff M, Davis RL, et al. Real-time vaccine safety surveillance for the early detection of adverse events. Med Care. 2007;45:S89-95.

39. Cook AJ, Tiwari RC, Wellman RD, et al. Statistical approaches to group sequential monitoring of postmarket safety surveillance data: current state of the art for use in the Mini-Sentinel pilot. Pharmacoepidemiol Drug Saf. 2012;21(Suppl 1):72-81.

40. Nelson JC, Cook AJ, Yu O, et al. Challenges in the design and analysis of sequentially monitored postmarket safety surveillance evaluations using electronic observational health care data. Pharmacoepidemiol Drug Saf. 2012;21(Suppl 1):62-71.

41. Gee J, Naleway A, Shui I, et al. Monitoring the safety of quadrivalent human papillomavirus vaccine: findings from the Vaccine Safety Datalink. Vaccine. 2011;29:8279-84.
42. Gagne J, Wang S, Schneeweiss S. Mini-Sentinel prospective routine observational monitoring program tool: cohort matching. 2014; http://www.mini-sentinel.org/work_products/Statistical_ Methods/Mini-Sentinel_PROMPT_Cohort-Matching-Tool_Technical-Users-Guide.pdf. Accessed 30 Jan 2014.

43. Carnahan R, Gagne J, Nelson J, et al. Mini-Sentinel Prospective Routine Observational Monitoring Program Tools (PROMPT): Rivaroxaban Surveillance Plan. 2014; http://www.mini-sentinel. org/work_products/Assessments/Mini-Sentinel_PROMPT_Riva roxaban-Surveillance-Plan.pdf. Accessed 19 Feb 2014.

44. Buggy Y, Cornelius V, Wilton L, Shakir SA. Risk of depressive episodes with rimonabant: a before and after modified prescription event monitoring study conducted in England. Drug Saf. 2011;34:501-9.

45. Trifirò $\mathrm{G}$, Coloma $\mathrm{P}$, Romio $\mathrm{S}$, et al. Risk of cardiac valve disorders associated with the use of biphosphonates. Final protocol of a signal strengthening study. 2012; http://www.encepp.eu/ encepp/openAttachment/fullProtocol/2615. Accessed 15 Jan 2014.

46. Dormuth CR, Hemmelgarn BR, Paterson JM, et al. Use of high potency statins and rates of admission for acute kidney injury: multicenter, retrospective observational analysis of administrative databases. BMJ. 2013;346:f880.

47. Filion KB, Chateau D, Targownik LE, et al. Proton pump inhibitors and the risk of hospitalisation for community-acquired pneumonia: replicated cohort studies with meta-analysis. Gut. 2013;63:552-8.

48. Glanz JM, McClure DL, Xu S, et al. Four different study designs to evaluate vaccine safety were equally validated with contrasting limitations. J Clin Epidemiol. 2006;59:808-18.

49. Yih WK, Lieu TA, Kulldorff M, et al. Intussusception risk after rotavirus vaccination in U.S. infants. N Engl J Med. 2014;370: 503-12.

50. FDA safety communication: FDA releases final study results of a Mini-Sentinel postlicensure observational study of rotavirus vaccines and intussusception. 2013; http://www.fda.gov/Biologics BloodVaccines/SafetyAvailability/ucm356758.htm. Accessed 26 Jan 2014.

51. Narcolepsy in association with pandemic influenza vaccination. A multi-country European epidemiological investigation. Stockholm: European Centre for Disease, Prevention and Control; 2012.

52. EMA. Questions and answers on the review of Pandemrix influenza vaccine (H1N1) (split virion, inactivated, adjuvanted) A/California/7/2009 (H1N1)v like strain (X-179A) 2011; http:// www.ema.europa.eu/docs/en_GB/document_library/Medicine_ QA/2011/07/WC500109183.pdf. Accessed 21 Jan 2014.

53. EMA. European Medicines Agency reviews hypothesis on Pandemrix and development of narcolepsy. 2012; http://www.ema. europa.eu/docs/en_GB/document_library/Press_release/2012/10/ WC500134087.pdf. Accessed 16 Jan 2014.

54. Cutrona SL, Toh S, Iyer A, et al. Design for validation of acute myocardial infarction cases in Mini-Sentinel. Pharmacoepidemiol Drug Saf. 2012;21(Suppl 1):274-81.

55. Walsh KE, Cutrona SL, Foy S, et al. Validation of anaphylaxis in the Food and Drug Administration's Mini-Sentinel. Pharmacoepidemiol Drug Saf. 2013;22:1205-13.

56. Lo Re V 3rd, Haynes K, Goldberg D, et al. Validity of diagnostic codes to identify cases of severe acute liver injury in the US Food and Drug Administration's Mini-Sentinel Distributed Database. Pharmacoepidemiol Drug Saf. 2013;22:861-72.

57. Herrett E, Thomas SL, Schoonen WM, et al. Validation and validity of diagnoses in the General Practice Research Database: a systematic review. Br J Clin Pharmacol. 2010;69:4-14.

58. Coloma PM, Valkhoff VE, Mazzaglia G, et al. Identification of acute myocardial infarction from electronic healthcare records 
using different disease coding systems: a validation study in three European countries. BMJ Open. 2013;3:e002862.

59. Zhou X, Murugesan S, Bhullar $\mathrm{H}$, et al. An evaluation of the THIN database in the OMOP Common Data Model for active drug safety surveillance. Drug Saf. 2013;36:119-34.

60. EU-ADR website. 2012; http://www.eu-adr-project.com/drupal/ ?q=home. Accessed 18 Dec 2013.

61. Detection and analysis of adverse events related to regulated products in automated healthcare data: efforts to develop the Sentinel Initiative. http://www.fbo.gov/index?s=opportunity\&mode =form \&id=91c24d08cb29861e15bca1b2062b5704\&tab=core\&_ cview $=1$. Accessed 31 Jan 2014.

62. The New Zealand Pharmacovigilance Centre-Intensive Medicines Monitoring Programme: Funding Statement. https://nzphvc. otago.ac.nz/immp/?page_id=21. Accessed 31 Jan 2014.

63. Coloma PM, Trifiro G, Patadia V, Sturkenboom M. Postmarketing safety surveillance: where does signal detection using electronic healthcare records fit into the big picture? Drug Saf. 2013;36:183-97.

64. New approaches to strengthen pharmacovigilance. Drug Discov Today Technol. 2011;8:e1-42.

65. Williams MP, Buchbinder R, March L, Lassere M. The Australian Rheumatology Association Database (ARAD). Semin Arthritis Rheum. 2011;40:e2-3.

66. Hernandez-Diaz S, Smith CR, Shen A, et al. Comparative safety of antiepileptic drugs during pregnancy. Neurology. 2012;78: 1692-9.

67. Praditpornsilpa K, Tiranathanagul K, Kupatawintu P, et al. Biosimilar recombinant human erythropoietin induces the production of neutralizing antibodies. Kidney Int. 2011;80:88-92.

68. OHDSI (Observational Health Data Sciences and Informatics). 2013; http://www.ohdsi.org/. Accessed 28 May 2014.

69. Nakashima N. Japanese sentinel project and contribution of laboratory medicine. Rinsho Byori. 2013;61:501-10.

70. The European Network of Centres for Pharmacoepidemiology and Pharmacovigilance (ENCePP). Guide on methodological standards in pharmacoepidemiology (revision 2). EMA/95098/ 2010; http://www.encepp.eu/standards_and_guidances. Accessed 10 Feb 2014.

71. Chen RT, Glasser JW, Rhodes PH, et al. Vaccine Safety Datalink project: a new tool for improving vaccine safety monitoring in the
United States. The Vaccine Safety Datalink Team. Pediatrics. 1997;99:765-73.

72. Coloma PM, Schuemie MJ, Trifiro G, et al. Combining electronic healthcare databases in Europe to allow for large-scale drug safety monitoring: the EU-ADR Project. Pharmacoepidemiol Drug Saf. 2011;20:1-11.

73. Prieto-Alhambra D. Information System for the Development of Primary Care Research. 2013; http://www.sidiap.org/images/ stories/docs/SIDIAP_angles_v2.pdf. Accessed 30 Jan 2014.

74. Parkinson J. Clinical Practice Research Datalink. 2012; http:// www.scot-ship.ac.uk/sites/default/files/Retreat/John_Parkinson. pdf. Accessed 1 Feb 2014.

75. Curtis LH, Weiner MG, Boudreau DM, et al. Design considerations, architecture, and use of the Mini-Sentinel distributed data system. Pharmacoepidemiol Drug Saf. 2012;21(Suppl 1):23-31.

76. Hoyle T, McMahill-Walraven C, Selvam N, et al. Equippin PRISM for pandemic influenza interoperability specification for data partners and immunization registries. 2013; http://www. mini-sentinel.org/work_products/PRISM/Mini-Sentinel_PRISM_ Equipping-PRISM-for-Pandemic-Influenza_Interoperability-Spec ification.pdf. Accessed 18 Dec 2013.

77. Racoosin J. A national strategy for monitoring medical product safety. FDA's Sentinel Initiative 2010; http://www.patientnetwork.fda.gov/sites/default/files/072910_sentinelinitiative.pdf. Accessed 11 Dec 2013.

78. Asian Pharmacoepidemiology Network. "Modified" Distributed Network Model. 2012; http://aspennet.asia/pdf/modified_distributed_network_model.pdf. Accessed 20 Dec 2013.

79. Lawson DH, Sherman V, Hollowell J. The general practice research database. Scientific and Ethical Advisory Group. QJM. 1998;91:445-52.

80. Zornberg GL, Hsu L, Dong D, et al. Dronedarone or amiodarone and risk of heart failure: a Federal Partners Collaboration. Abstract presented at 27th International Conference on Pharmacoepidemiology and Therapeutics, Chicago, IL; 2011.

81. Pratt N, Andersen M, Bergman U, et al. Multi-country rapid adverse drug event assessment: the Asian Pharmacoepidemiology Network (AsPEN) antipsychotic and acute hyperglycaemia study. Pharmacoepidemiol Drug Saf. 2013;22:915-24. 